\title{
Estudios preliminares sobre congelación de embriones de yamú (Brycon amazonicus) en diferentes estadios de desarrollo
}

\author{
Preliminary studies on embryo freezing of yamú \\ (Brycon amazonicus) in different stages of development
}

\section{Estudos preliminares sobre congelamento de embriões do matrinxã (Brycon amazonicus) em diferentes estágios de desenvolvimento}

\author{
Alexander Torres-Tabares ${ }^{1 *}$; Nicolás Novoa-Rodríguez ${ }^{2}$; Leydy Y. Sandoval-Vargas ${ }^{3 *}$; \\ Yohana M. Velasco-Santamaría ${ }^{4 *}$; Pablo E. Cruz-Casallas ${ }^{5 *}$; Víctor M. Medina-Robles ${ }^{6 *}$
}

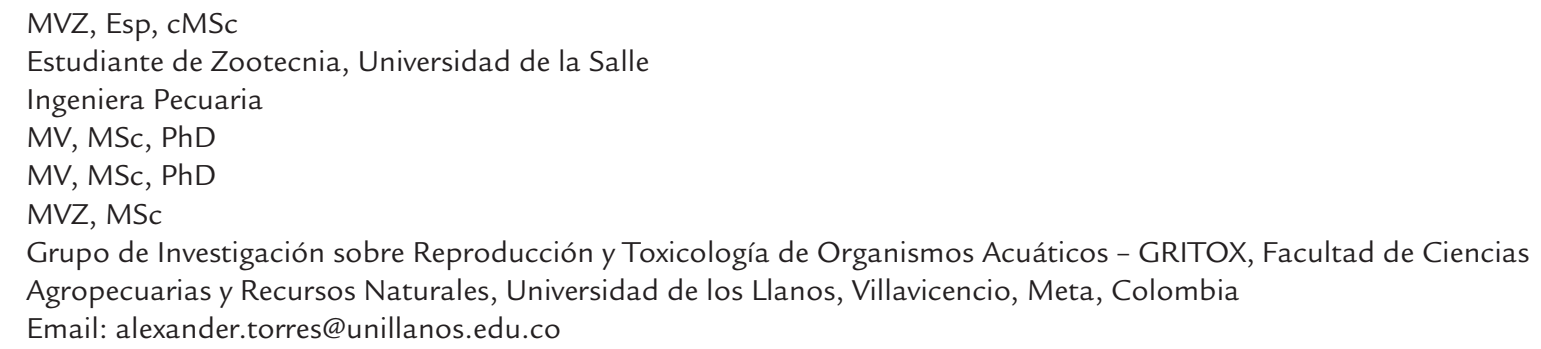

Recibido: septiembre 4 de $2012 \quad$ Aceptado: noviembre 29 de 2012

\begin{abstract}
Resumen
La estacionalidad reproductiva de las especies de peces tropicales ha limitado su cultivo comercial, debido a que limita la disponibilidad de alevinos. Una alternativa para contribuir a solucionar este problema es la crioconservación de gametos, ya que esta tecnología facilita su reproducción en cualquier época del año. Este estudio evaluó la viabilidad de embriones de yamú (Brycon amazonicus) conservados a $-14{ }^{\circ} \mathrm{C}$, como posible estrategia para el mejoramiento de su producción. Para este propósito, reproductores sexualmente maduros fueron inducidos a la reproducción con extracto de hipófisis de carpa [hembras $0.25(0 \mathrm{~h}), 0.5(24 \mathrm{~h})$ y $5.0(36 \mathrm{~h}) \mathrm{mg} / \mathrm{kg}$ y machos $(4.0 \mathrm{mg} / \mathrm{kg})]$. Los embriones resultantes, en estadio de blastulación (2 horas post-fertilización-hpf), gastrulación (6 hpf) y segmentación (8 hpf), fueron almacenados en tubos Falcon a $-14{ }^{\circ} \mathrm{C}$ previa refrigeración $\left(6{ }^{\circ} \mathrm{C}\right.$ durante $\left.10 \mathrm{~min}\right)$, suspendidos $(1: 1)$ en una solución de 10 y $15 \%$ de dimetilsulfóxido (DMSO) o metanol (MET). Transcurrida una hora de almacenamiento, los embriones fueron sumergidos en baño de agua a $27^{\circ} \mathrm{C}$ por 10 min y luego transferidos nuevamente a la incubadora, para evaluar su viabilidad y eclosión. La viabilidad y eclosión dependieron del estadio de desarrollo embrionario, así como del tipo de solución en que fueron almacenados. Los embriones del grupo control mostraron viabilidad y eclosión promedio de $96.2 \pm 0.1 \%$ y $96.3 \pm 0.7 \%$, respectivamente. Ninguno de los tratamientos que utilizó embriones de 2 hpf presentó eclosión; en contraste, embriones de $8 \mathrm{hpf}$ suspendidos en solución con 15\% de MET mostraron los mayores porcentajes de eclosión $(54.1 \pm 4.1 \%)$. En conclusión, embriones de yamú en estadios tardíos de desarrollo son susceptibles de conservarse a -14 ${ }^{\circ} \mathrm{C}$ por lo menos durante una hora.
\end{abstract}

Palabras clave: congelación, crioprotectores, desarrollo embrionario, eclosión, viabilidad, yamú. 


\begin{abstract}
Reproductive seasonality in fish has limited its commercial farming production due to the restriction in the fingerling availability. The cryopreservation is an alternative to solve this problem since facilitates the fish reproduction throughout the year. Therefore, this study evaluated the yamú embryos (Brycon amazonicus) viability conserved at $-14{ }^{\circ} \mathrm{C}$ as a possible strategy to improve its production. For this purpose, sexually mature broodstocks were induced to reproduction with carp pituitary extract [females $0.25(0 \mathrm{~h}), 0.5(24 \mathrm{~h})$ and $5(36 \mathrm{~h}) \mathrm{mg} / \mathrm{kg}$ and males $(4 \mathrm{mg} / \mathrm{kg})$ ]. The embryos in different stages of embryonic development such as blastulation (2 hours post-fertilization, hpf), gastrulation ( $6 \mathrm{hpf}$ ) and segmentation (8 hpf) were kept at $6{ }^{\circ} \mathrm{C}$ during $10 \mathrm{~min}$ and then storaged in falcon tubes at $-14{ }^{\circ} \mathrm{C}$ and subjected $(1: 1)$ in a solution of 10 and $15 \%$ of dimethyl sulfoxide (DMSO) or methanol (MET). After one hour of storage, the embryos were immersed in a water bath at $27{ }^{\circ} \mathrm{C}$ for $10 \mathrm{~min}$ and then transferred again to the incubators to evaluate viability and hatching. The viability and hatching depended to the embryonic development stage as well as the storage solution type. The embryos of the control group showed an average viability and hatching of $96.2 \pm 0.1$ and $96.3 \pm 0.7 \%$, respectively. No hatching was observed in the treatments with $2 \mathrm{hpf}$ embryos; in contrast, 8-hpf embryos subjected to MET 15\% showed the best hatching results $(54.1 \pm 4.1 \%)$. In conclusion, yamú embryos in later stages of development are susceptible to preserved at $-14{ }^{\circ} \mathrm{C}$ at least for 1 hour.
\end{abstract}

Key words: freezing, cryoprotectants, embryonic development, hatching, viability, yamú.

\title{
Resumo
}

A sazonalidade reprodutiva das espécies de peixes tropicais tem limitado o seu cultivo comercial, porque limita a disponibilidade de alevinos. Uma alternativa para ajudar a resolver este problema é a criopreservação de gametas, ao facilitar a reprodução em qualquer época do ano. Este estudo avaliou a viabilidade de embriões de matrinxã (Brycon amazonicus), mantidos a $-14{ }^{\circ} \mathrm{C}$, como uma possível estratégia para melhorar a produção de alevinos ao longo do ano. Para este efeito, reprodutores sexualmente maduros foram induzidos à reprodução com extrato de pituitária de carpa [fêmeas 0.25 ( 0 h), $0.5(24 \mathrm{~h})$ e $5.0(36 \mathrm{~h}) \mathrm{mg} / \mathrm{kg}$ e machos $(4 \mathrm{mg} / \mathrm{kg})]$. Embriões resultantes desta reprodução, na fase de blástula (2 horas pós-fertilização - hpf), gástrula (6 hpf) e segmentação (8 hpf), foram armazenados em tubos Falcon a $-14{ }^{\circ} \mathrm{C}$ depois de resfriamento $\left(6^{\circ} \mathrm{C}\right)$ durante $10 \mathrm{~min}$, suspensos $(1: 1)$ numa solução de 10 ou $15 \%$ de dimetil sulfóxido (DMSO) ou de metanol (MET). Após uma hora de armazenamento, os embriões foram submergidos num banho de água a $27^{\circ} \mathrm{C}$ durante 10 min e em seguida transferidos de novo para a incubadora, para avaliar a sua viabilidade e a eclosão. A viabilidade e a eclosão foram dependentes do estagio de desenvolvimento do embrião, bem como da solução de armazenamento utilizada. Os embriões do grupo controle mostraram viabilidade e eclosão em média de $96.2 \pm 0.1 \%$ e $96.3 \pm 0.7 \%$, respectivamente. Nenhum dos tratamentos que utilizaram embriões de 2 hpf apresentaram eclosão, em contraste, embriões de 8 hpf suspensos em solução de MET 15\% apresentaram os maiores percentagens de eclosão (54,1 $\pm 4,1 \%)$. Em conclusão, os embriões de matrinxã em estágios tardios do desenvolvimento são susceptíveis de ser armazenados a $-14{ }^{\circ} \mathrm{C}$, durante pelo menos uma hora.

Palavras-chave: congelamento, crioprotetores, desenvolvimento embrionário, eclosão, matrinxã, viabilidade.

\section{Introducción}

La piscicultura es una de las principales alternativas de producción del sector pecuario con excelentes perspectivas comerciales y productivas para la región de la Orinoquia Colombiana (Cruz-Casallas et al., 2006b); sin embargo, debido al aumento de la demanda de productos piscícolas y a la intensificación de los sistemas de producción, es necesario buscar estrategias para optimizar la eficiencia de los actuales procesos productivos.

Uno de los mayores problemas del cultivo de especies nativas de alto valor comercial es su marcada estacionalidad reproductiva, la cual limita la producción permanente de alevinos, generando una considerable variación en la oferta y demanda de sus productos. Con el fin de contribuir a la solución de esta problemática, es necesario utilizar estrategias que permitan garantizar la producción de alevinos durante todo el año para satisfacer la demanda de especies ícticas con alto potencial productivo como el yamú (Brycon amazonicus) (Velasco-Santamaría et al, 2006b).

La crioconservación es una técnica que permite conservar gametos, órganos y tejidos a temperaturas que detienen el metabolismo de sus células, pero conservando la viabilidad por un periodo de tiempo indeterminado. Para la crioconservación de gametos y embriones es necesario utilizar agentes crioprotectores, compuestos orgánicos responsables de proteger las estructuras y membranas celulares, al facilitar la deshidratación celular y por lo tanto reducir la formación de cristales de hielo intracelular (Niemann, 1991).

Durante los últimos años se han realizado en peces varios estudios enfocados a investigar diferentes metodologías para la crioconservación de gametos masculinos. En especies como yamú (Brycon amazonicus) 
y cachama blanca (Piaractus brachypomus) ya existen protocolos estandarizados para crioconservar células espermáticas a temperaturas criogénicas $\left(-196{ }^{\circ} \mathrm{C}\right)$ (Cruz-Casallas et al., 2006a; Velasco-Santamaría et al., 2006a; Medina-Robles et al., 2007); sin embargo, aún no existen para el yamú reportes sobre crioconservación exitosa de óvulos ni de embriones. En trabajos realizados en cachama blanca ( $P$. brachypomus) se ha obtenido viabilidad de casi $95 \%$ en embriones de 6 y 10 horas pos-fertilización, utilizando como crioprotector metanol a concentraciones del 10 y $15 \%$ y temperatura de conservación de $-14{ }^{\circ} \mathrm{C}$ (Castillo-Losada et al., 2011). Tecnologías como esta podrían convertirse en una herramienta útil, para conservar especies en potencial riesgo de extinción, además de extender el actual periodo de oferta de alevinos y de larvas (Cerdá, 2002). Por lo anterior, el objetivo del presente estudio fue evaluar la viabilidad de embriones de yamú (B. amazonicus) de diferentes estadios de desarrollo, conservados a $-14{ }^{\circ} \mathrm{C}$ durante 1 hora y suspendidos en dos soluciones crioprotectoras, como posible estrategia para el mejoramiento de su producción.

\section{Materiales y métodos}

\section{Comité de ética}

Todos los procedimientos fueron realizados de conformidad con las normas y procedimientos para el uso de animales de laboratorio, descritas por el Comite on Care and Use of Laboratory Animal Resources - National Research Council, USA (1996). De igual forma, los procedimientos del presente estudio contaron con la aprobación del Comité de Bioética de la Universidad de los Llanos.

\section{Localización}

El trabajo fue llevado a cabo en el Laboratorio de Reproducción y Crioconservación de Gametos del Instituto de Acuicultura de la Universidad de los Llanos (IALL-UNILLANOS), ubicado en la ciudad de Villavicencio-Meta (Colombia), a una altura de $418 \mathrm{msnm}$, temperatura promedio de $26{ }^{\circ} \mathrm{C}$, humedad relativa del $75 \%$ y precipitación pluvial de $4.050 \mathrm{~mm}$.

\section{Material biológico}

Se utilizaron reproductores de yamú (B. amazonicus) clínicamente sanos, sexualmente maduros con peso corporal de $1.2 \pm 0.2 \mathrm{~kg}$ y talla de $44 \pm 1 \mathrm{~cm}$ de longitud total, provenientes de la estación piscícola de la Universidad de los Llanos.
La condición reproductiva de las hembras fue evaluada mediante biopsia ovárica, realizada para determinar el desarrollo de los oocitos y la migración de sus núcleos. En los machos se evaluó la presencia de semen en la papila urogenital después de realizar masaje en sentido cráneo-caudal del abdomen. Luego de seleccionar los peces, estos se identificaron por medio de códigos de colores ubicados en la aleta dorsal.

\section{Calidad del agua}

Para el proceso de incubación se utilizó un sistema de recirculación con filtro de arena, alimentado con agua extraída de un pozo profundo de la Estación Piscícola de la Universidad de los Llanos. Previamente el agua de incubación fue sometida a un proceso de maduración y sus características físicas y químicas ajustadas a $\mathrm{pH}$ 6.5-7.0, temperatura $24-28{ }^{\circ} \mathrm{C}$ y oxígeno disuelto 4-6 mg/l. Cada seis horas fueron evaluados las características físicas y químicas del agua, como temperatura $\left({ }^{\circ} \mathrm{C}\right)$, oxígeno disuelto $(\mathrm{mg} / \mathrm{l})$ y $\mathrm{pH}$, por medio de una sonda multiparamétrica (YSI MPS 556, Yellow Spring Instruments); de igual modo, se determinó la dureza, alcalinidad, nitratos y nitritos $(\mathrm{mg} / \mathrm{l})$ por medio de un kit de calidad de aguas (Aquamerck, Merck).

\section{Protocolo de inducción hormonal}

La maduración final de las gónadas se indujo mediante aplicación intramuscular de Extracto de Hipófisis de Carpa - EHC (Stoller Fisheries, USA) distribuida en tres aplicaciones [0.25 (24h), 0.5 (12h) y 5 (36h) mg/kg]. La inducción hormonal de los machos se realizó mediante una única inyección equivalente a $4 \mathrm{mg} / \mathrm{kg}$ de peso corporal, aplicada simultáneamente con la segunda dosis de tratamiento de las hembras.

\section{Obtención de gametos}

La obtención de los gametos se realizó aproximadamente $6 \mathrm{~h}\left(27^{\circ} \mathrm{C}\right)$ después de la última dosis hormonal en la hembra; para lo cual los animales fueron tranquilizados con 2-fenoxietanol $(300 \mathrm{mg} / \mathrm{l}$, Sigma Chemical Co, St. Louis, Missouri, USA) hasta la pérdida del eje de nado. Posteriormente, los peces fueron removidos de la solución anestésica y se realizó un masaje cráneo-caudal del abdomen para la obtención de los oocitos y del semen, los cuales fueron colectados en un recipiente plástico y tubos de vidrio aforados, respectivamente. Finalmente, se realizó la fertilización e hidratación de los ovocitos, que luego fueron transferidos a una incubadora tipo Woynarovich de flujo ascendente de 2001 . 


\section{Características de selección embrionaria}

Antes de la congelación fueron evaluados los estadios de desarrollo embrionario para cada tratamiento, observándose que los embriones de 2 hpf se encontraban en periodo de blastulación, caracterizado por descenso y formación del blastodisco sobre el vitelo insegmentado. Así mismo, los embriones de 6 hpf se encontraron en estadio de gastrulación caracterizados por epibolia del $90 \%$ donde se formó el tapón vitelino y cierre del blastoporo, haciéndose más evidente la diferenciación del eje cráneo-caudal. Finalmente, a las 8 hpf el estadio de desarrollo correspondió al periodo de segmentación y faringulación, en esta etapa se observó un embrión con diferenciación antero-posterior, con simetría bilateral, dispuesto sobre el vitelo tomando una forma arqueada inicial donde se observaron claramente las somitas y se diferenció la vesícula óptica y ótica (Figura 4).

\section{Crioprotectores}

Se emplearon dos crioprotectores permeables: metanol (MET, Merck KGaA, Darmstadt, Germany) y dimetilsulfóxido (DMSO, Sigma Chemical Co, St. Louis, USA), en combinación con un crioprotector no permeable o externo (glucosa, Merck). Las concentraciones evaluadas fueron 10 y $15 \%$ para DMSO y MET cada uno con el $20 \%$ de glucosa. Todos los diluyentes fueron disueltos en solución salina fisiológica ( $\mathrm{NaCl}$ 0,9\%).

\section{Diseño experimental}

Se realizaron 12 tratamientos, constituidos por dos soluciones crioprotectoras (dimetil-sulfóxido y metanol), cada uno con dos concentraciones (10 y 15\%) en tres estadios de desarrollo embrionario (blastulación, gastrulación y segmentación, correspondientes a las 2, 4 y 8 horas post-fertilización, respectivamente). Se reali-

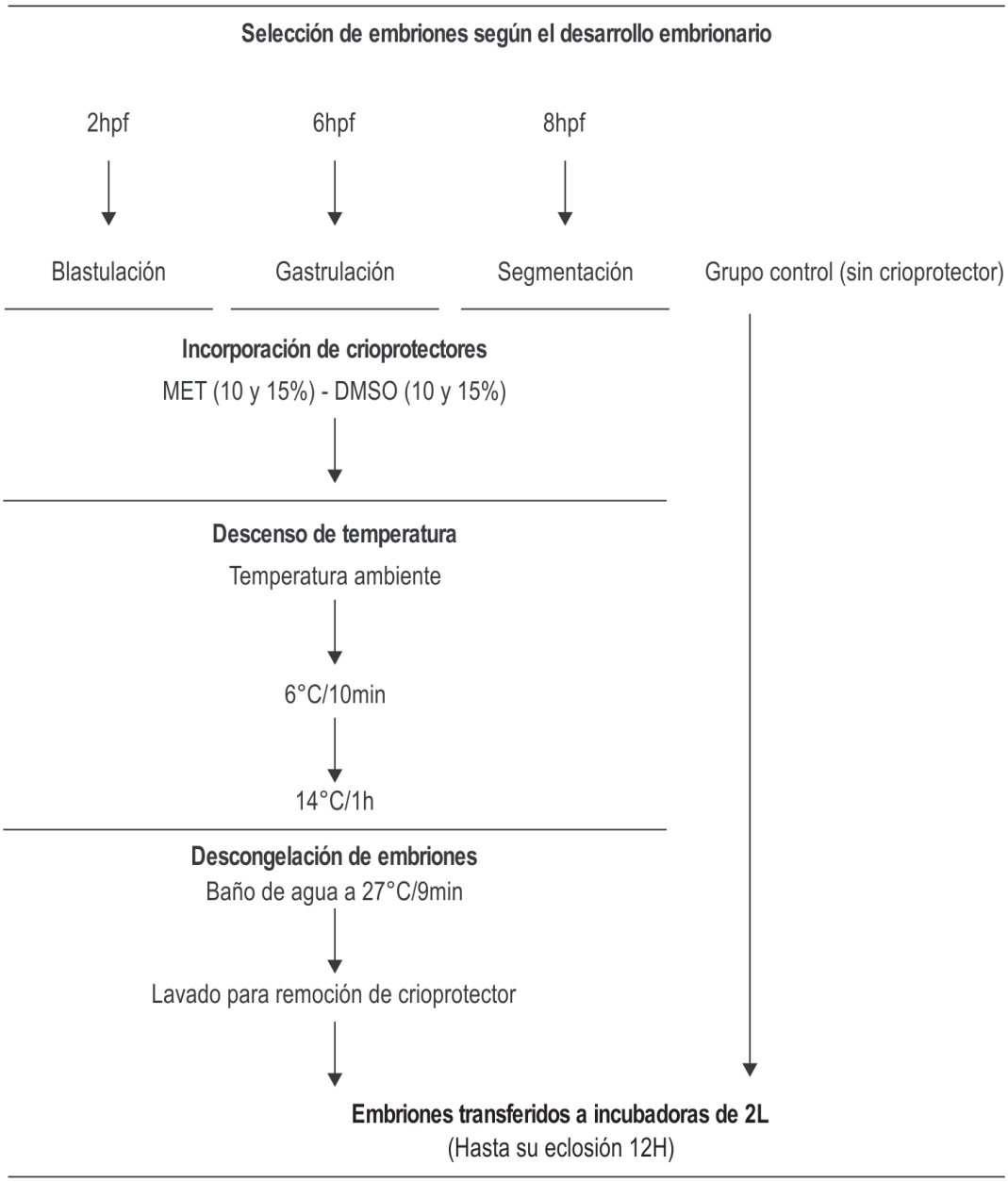

Figura 1. Protocolo de congelación de embriones de yamú (B. amazonicus) durante diferentes estadios del desarrollo embrionario sometidos a $-14^{\circ} \mathrm{C}$ durante $1 \mathrm{~h}$. 
zaron cuatro réplicas para cada tratamiento cada una con su respectivo grupo control, siendo estos aquellos embriones no sometidos a congelación (Figura 1).

\section{Congelación y descongelación de embriones}

Transcurridas las hpf para cada uno de los estadios de desarrollo embrionario a evaluar (2h blastulación, $6 \mathrm{~h}$ gastrulación y 8 h segmentación-faringulación) fueron tomadas muestras de $6 \mathrm{ml}$ de embriones para cada tratamiento y seguidamente depositados en tubos plásticos tipo Falcon estériles de $13 \mathrm{ml}$. A cada una de las muestras se le adicionó $6 \mathrm{ml}$ del diluyente correspondiente con MET o DMSO a concentraciones de 10 y $15 \%$, en una proporción de $6 \mathrm{ml}$ de embriones y 6 $\mathrm{ml}$ de diluyente $(1: 1)$. Con el fin de permitir un tiempo de equilibrio entre los embriones y cada tratamiento, cada tubo falcon fue llevado a una cabina de refrigeración a $6{ }^{\circ} \mathrm{C}$ durante $10 \mathrm{~min}$, trascurrido este tiempo las muestras se llevaron a un congelador digital programable (Samsung RS20 - 555L, USA) donde el descenso de temperatura fue aproximadamente de $3{ }^{\circ} \mathrm{C} /$ min, posteriormente, permanecieron a temperatura de $-14{ }^{\circ} \mathrm{C}$ durante 1 hora hasta su descongelación.

Las muestras fueron descongeladas por inmersión directa durante 9 minutos en baño de agua a $27^{\circ} \mathrm{C}$, de acuerdo con lo descrito por Castillo-Losada et al. (2011). Este sistema ofrece una tasa de descongelación rápida evitando procesos de recristalización celular. Los embriones descongelados de cada tratamiento fueron lavados con agua de incubación para eliminar los residuos del crioprotector y posteriormente fueron ubicados en incubadoras experimentales de flujo ascendente con capacidad de $2 \mathrm{I}$.

\section{Viabilidad del desarrollo embrionario}

Los tratamientos se evaluaron cada dos horas a partir de la descongelación e iniciada la incubación artificial de los embriones, tomando muestras representativas de embriones ( 50) mediante varillas de vidrio, para determinar el porcentaje de embriones viables (traslúcidos-viables y blancos- inviables) hasta su eclosión. Así mismo, se verificaron los resultados mediante observaciones realizadas por medio de un estéreo microscopio (Nikon SMZ 800, Japón).

\section{Determinación de eclosión larval}

La eclosión fue cuantificada tomando tres muestras representativas de cada réplica y promediando el número de larvas eclosionadas (rompimiento del corion), expresando el resultado en porcentaje (\%). Las obser- vaciones fueron realizadas dependiendo del estado de desarrollo embrionario así: los embriones de $2 \mathrm{hpf}$ estado de blastulación eclosionaron a las 10 horas de incubación, los de 6 hpf estado de gastrulación eclosionaron a las 6 horas de incubación y los embriones de 8 hpf estado de segmentación faringulación eclosionaron a las 4 horas de incubación. Este tiempo fue establecido teniendo en cuenta que la eclosión de larvas de yamú tarda aproximadamente 12.5 horas a una temperatura de $27^{\circ} \mathrm{C}$ (Arias et al., 2006). Las larvas que presentaron características atípicas como color blan$\mathrm{Co}$, sin movimiento y desarrollo incompleto no fueron consideradas para determinar eclosión larval.

\section{Evaluación de la morfología pre y post congelación}

Con el fin de determinar los posibles cambios morfológicos generados durante el proceso de conservación, muestras de embriones fueron registradas fotográficamente al inicio del experimento, luego de la aplicación del respectivo crioprotector (MET y DMSO al 10 y $15 \%$ cada uno) y posterior al proceso de descongelación. Estos registros fueron tomados mediante una cámara digital (Nikon Digital Sight, DS-5M, Japón) ajustada a un estereoscopio binocular (Nikon SMZ 800, Japón).

\section{Análisis estadístico}

Todos los valores fueron expresados como media \pm error estándar de la media (SEM). Los datos no cumplieron los supuestos de normalidad y homogeneidad de varianza evaluados mediante la prueba de KolmogorovSmirnov y Bartlett, respectivamente. Por tanto, con el fin de evaluar la interacción de cada factor y el efecto de cada tratamiento sobre las variables de viabilidad embrionaria y eclosión se empleó análisis factorial con los datos transformados en ranks, seguido de una prueba de comparación de medias Tukey. En todos los casos $p<0.05$ fue utilizado como criterio estadístico para revelar diferencias significativas. Los datos se analizaron con el software SAS versión 9.2 para Windows (2002-2008 por SAS Institute Inc, Cary, NC. USA).

\section{Resultados}

\section{Viabilidad embrionaria}

Se observaron diferencias estadísticas significativas en todos los estadios de desarrollo embrionario con respecto a los grupos control, los cuales mostraron viabilidad promedio de $96.2 \pm 0.09 \%(p<0.05)$ (Figura 2). Las mejores viabilidades fueron para los embriones en estado de gastrulación $31.7 \pm 6.0$, y segmentaciónfaringulación $69.5 \pm 2.8 \%$ utilizando como crioprotec- 
tor metanol 10 y $15 \%$, respectivamente (Figura 2). Los embriones en estado de blastulación (2 hpf) expuestos a los diferentes crioprotectores presentaron baja viabilidad con respecto a los demás estadios de desarrollo embrionario. Para esta etapa, solo se obtuvó viabilidad en los tratamientos expuestos a MET y DMSO $10 \%$ $(1.3 \pm 0.5$ y $0.5 \pm 0.3 \%$, respectivamente) (Figura 2$)$.

El aumento de la viabilidad fue directamente proporcional a la edad del embrión, obteniéndose en todos los casos mayores porcentajes en los periodos de segmentación-faringulación (8hpf), seguido por la etapa de gastrulación, correspondiente a la sexta hora de desarrollo embrionario; sin embargo, para esta última etapa, la viabilidad no superó el $31.7 \pm 6.0 \%$, la cual fue obtenida con el tratamiento de MET 10\% (Figura 2).

\section{Sobrevivencia larval}

No se obtuvó porcentaje de eclosión a las 2 hpf debido a la baja tasa de viabilidad observada en los tratamientos de MET y DMSO 10 y $15 \%$, por tanto, en la figura 3 sólo se ilustran los resultados de eclosión correspondientes a las 6 y 8 HPF.

La eclosión para estos últimos dos estados de desarrollo embrionario mostró un comportamiento similar al que venía presentando la variable de viabilidad, siendo el MET 10 y $15 \%$ los que nuevamente presentaron las mejores tasas de eclosión.

Como se puede apreciar en la figura 3, las mejores tasas de eclosión fueron obtenidas en el estado de segmentación-faringulación con $54.1 \pm 4.1 \%$ para MET $15 \%$ y en el estado de gastrulación con $26.8 \pm 7.6$ para MET $10 \%$, presentando diferencias significativas con respecto al control $(p<0.05)$, pero no entre tratamientos. Por su parte, las menores tasas de eclosión fueron observadas en todos los tratamientos con DMSO, el cual presentó diferencias significativas con respecto a los demás tratamientos $(\mathrm{p}<0.05)$ (Figura 3$)$.

\section{Evaluación pre-congelación con adición de crioprotector}

Los embriones en estado de blastulación (2 hpf), sometidos a los crioprotectores MET y DMSO 10 y $15 \%$ presentaron daño a nivel de la formación del blastodisco, en los cuales se observó alargamiento de esta estructura y pérdida de la forma circular del corion debido a la deshidratación; igualmente se presentó daño de la morfología normal del embrión (Figura 5). Por el contrario, los embriones en la etapa de gastrulación (6 hpf) no mostraron cambios evidentes a nivel estructural, pero si presentaron oscurecimiento del saco vitelino y formaciones de pliegues en el corion (Figura 6).

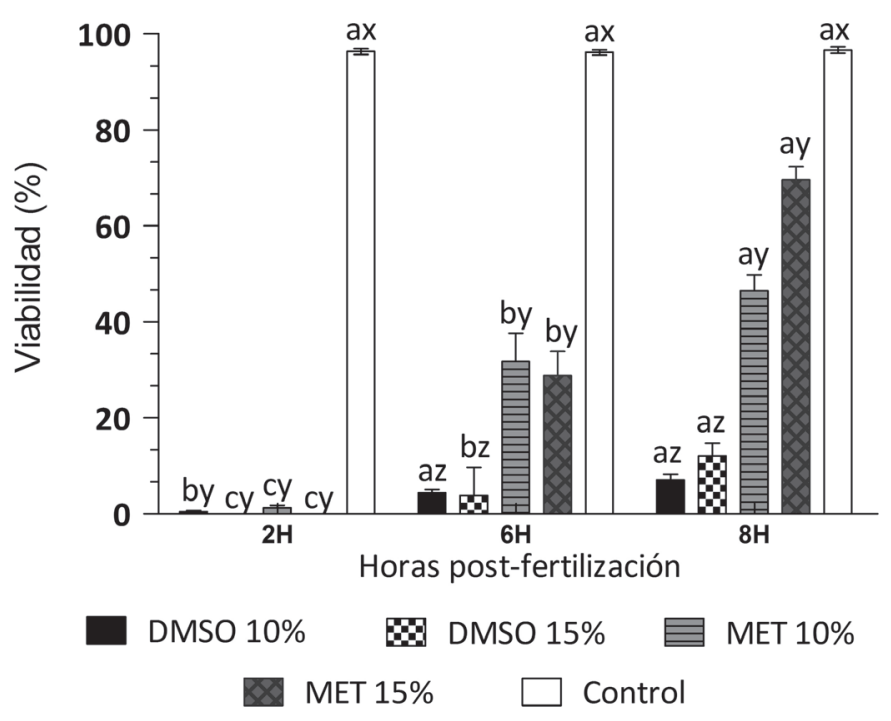

Figura 2. Tasa de viabilidad de embriones de yamú (B. amazonicus) en tres estadios del desarrollo embrionario conservados durante una hora a $-14{ }^{\circ} \mathrm{C}$ con diferentes concentraciones de dimetil-sulfóxido (DMSO) y metanol (MET).

a, b, c Para un mismo tratamiento a diferentes horas de post-fertilización, barras con letras diferentes difieren estadísticamente $(\mathrm{p}<0.05)$.

$x, y, z$ Dentro de una misma hora, barras con letras diferentes son estadísticamente diferentes con respecto al control ( $p<0.05$ ). Los valores son mostrados como media \pm SEM. 


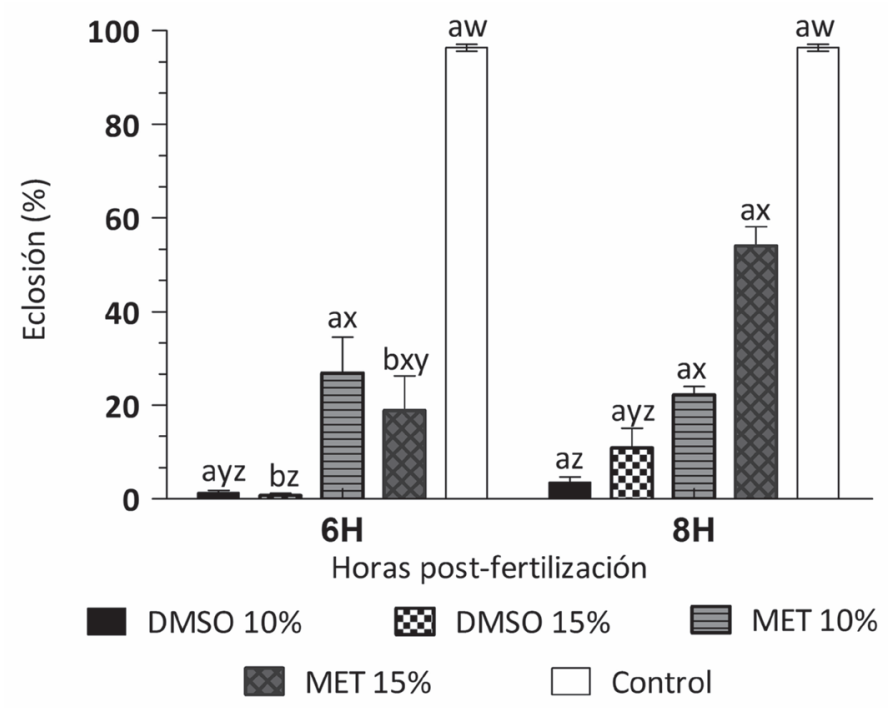

Figura 3. Tasa de eclosión de embriones de yamú (B. amazonicus) en tres estadios del desarrollo embrionario conservados durante una hora a $-14{ }^{\circ} \mathrm{C}$ con diferentes concentraciones de Dimetil-sulfóxido (DMSO) y Metanol (MET).

a, b, c Para un mismo tratamiento a diferentes horas de post-fertilización, barras con letras diferentes difieren estadísticamente $(\mathrm{p}<0.05)$.

$x, y, z$. Dentro de una misma hora, barras con letras diferentes son estadísticamente diferentes con respecto al control ( $p<0.05)$. Los valores son mostrados como media \pm SEM.

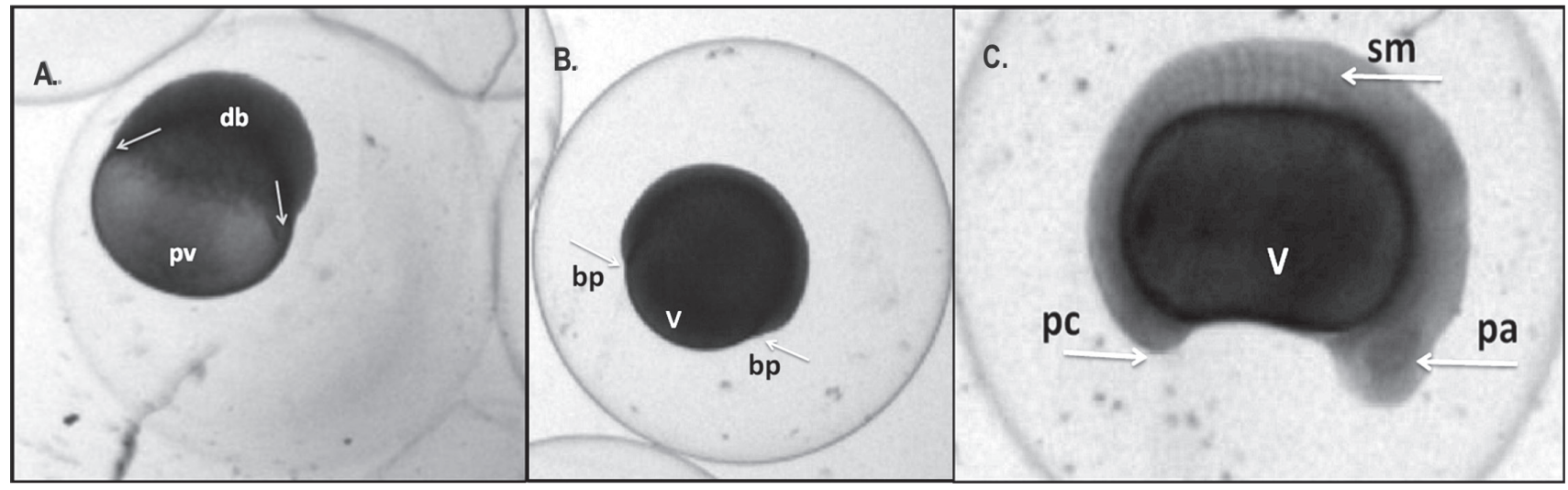

Figura 4. Desarrollo embrionario de yamú (B. amazonicus). A). 2 hpf inicio del descenso del discoblástula (db) sobre el polo vegetal (pv), B). 6 hpf epibólia del 90 \%, cierre del blastoporo, borde periblástico (bp) vitelo (v), C). 8 hpf embrión diferenciado anteroposterior en forma arqueada, somitas (sm), parte anterior (pa), parte caudal (pc).
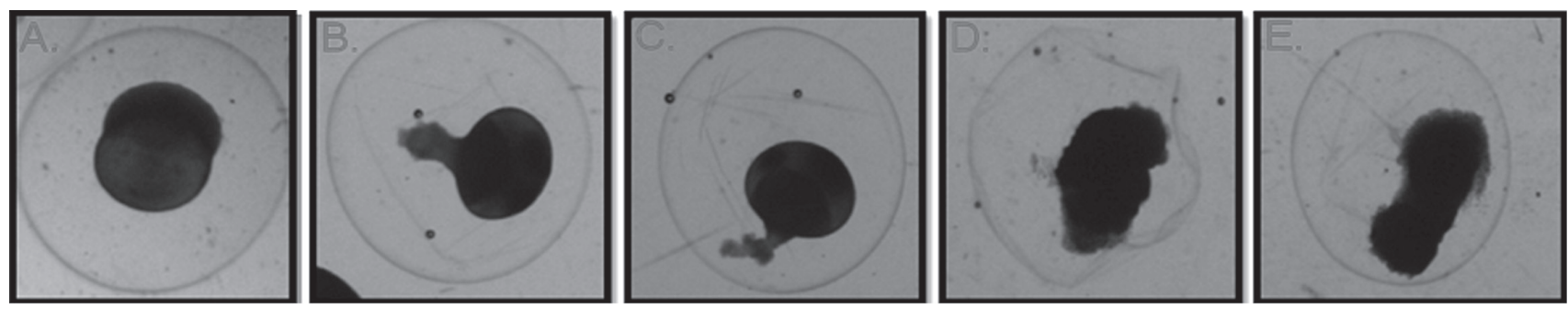

Figura 5. Embriones de yamú (B. amazonicus) en estado de blastulación (2 hpf) una vez expuestos a diferentes tratamientos con crioprotector. A) Grupo control, B) MET 10\%, C) MET 15 \%, D) DMSO 10\%, E) DMSO 15\%. 

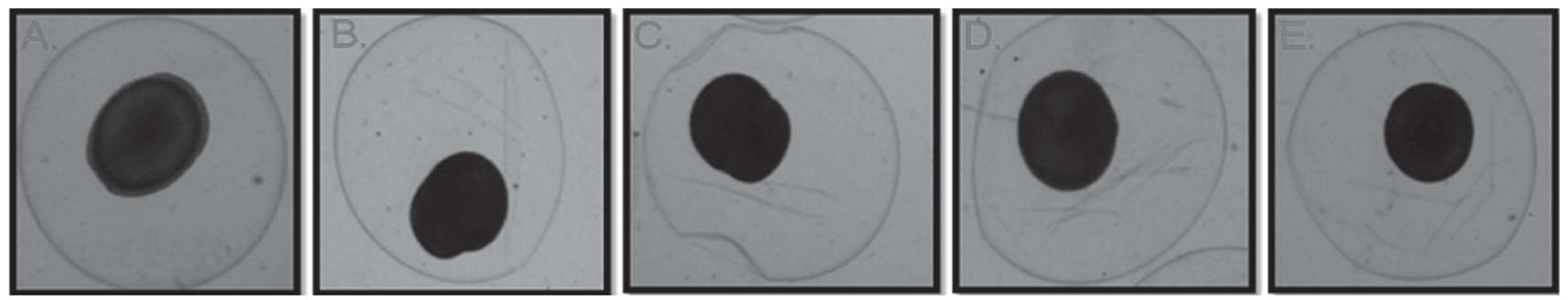

Figura 6. Embriones de yamú (B. amazonicus) en estado de gastrulación (6 hpf), una vez expuestos a diferentes tratamientos con crioprotector. A) Grupo control, B) MET 10\%, C) MET $15 \%$, D) DMSO 10\%, E) DMSO 15\%.

Por su parte, los embriones en estado de segmentación-faringulación (8 hpf), sometidos a DMSO (10 y $15 \%$ ) presentaron mayor daño a nivel morfológico comparados con los sometidos a MET en las mismas concentraciones, observándose daños estructurales a nivel cefálico, vesícula óptica, ótica y somitas. Los embriones expuestos a MET presentaron diferentes porcentajes de deshidratación del espacio perivitelinico con el correspondiente daño en las estructuras que conforman el corion (Figura 7).

\section{Evaluación post-congelación (1 Hora):}

En cuanto a los cambios morfológicos post-congelación, se observó que los embriones en estado de blastulación (2 hpf) sometidos a MET y DMSO (10 y 15\%) presentaron daño total a nivel estructural con destrucción del vitelo y cambio de coloración del corion (gris oscuro) (Figura 8). Por su parte, los embriones en etapa de gastrulación (6 hpf) presentaron desintegración de las estructuras perivitelinicas y deformación de la epibolia. Por otro lado, los embriones sometidos a MET $10 \%$ presentaron daño en la membrana vitelina y una reducción en su desarrollo mientras que los expuestos a MET $15 \%$ se evidenciaron cambios relevantes a nivel de la integridad de sus estructuras internas (Figura 9).

Por último, los embriones de $8 \mathrm{hpf}$ sometidos a las dos concentraciones de DMSO presentaron mayor daño a nivel morfológico comparado con el grupo control donde se observó indiferenciación a nivel antero-posterior y no hubo formación de vesículas ni saco vitelino. En los tratamientos expuestos a MET se presentaron los mejores resultados debido a que no se evidenciaron cambios morfológicos que limitaran el desarrollo normal de los embriones (Figura 10).

\section{Discusión}

En el mundo ya se encuentran estandarizados diferentes protocolos de congelación de embriones de diferentes especies, incluidos humanos (Kawayama et al., 2007), bovinos (Pryor et al., 2011) y ratones (Kasai et al., 2002), entre otras. A pesar de los resultados favorables que se han obtenido a nivel mundial con estas especies, en peces la crioconservación de embriones aún presenta algunos inconvenientes ocasionados principalmente por la baja permeabilidad de las membranas, la toxicidad de los crioprotectores y la formación de cristales de hielo, lo cual genera baja viabilidad y eclosión en los procesos de incubación posterior.

En el presente trabajo los resultados de viabilidad y eclosión estuvieron directamente relacionados con el estadio del desarrollo embrionario, siendo los embriones menos avanzados ( 2 hpf blastulación) los más susceptibles a los criodaños, tal como ha sido reportado por Zhang y Rawson (1995) y Zhang et al. (2003). Lo anterior se debe probablemente a que en especies de peces tropicales como el yamú (B. amazonicus), el desarrollo embrionario es rápido presentando seis estadios (ovocélula a las cero hpf; primer clivaje a las 0.6
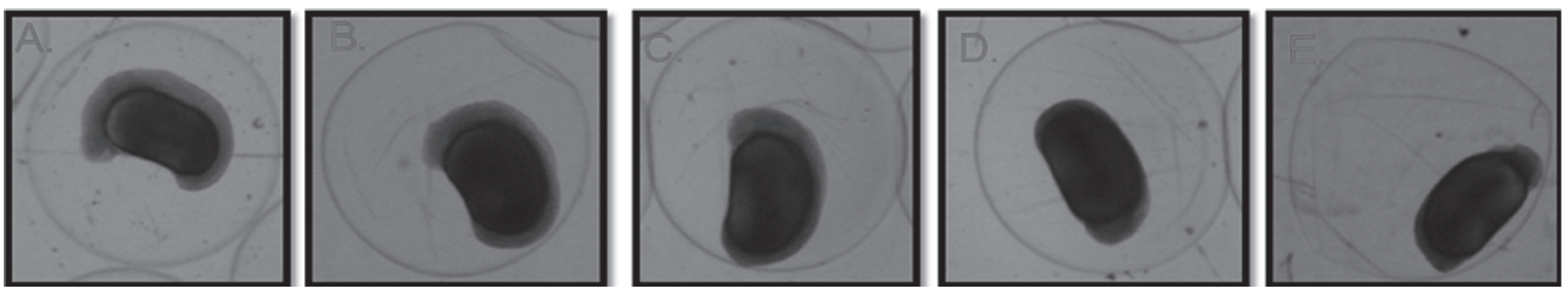

Figura 7. Embriones de Yamú (B. amazonicus) en estado de segmentación-faringulación (8 hpf) una vez expuestos a diferentes tratamientos con crioprotector. A) Grupo control, B) MET 10\%, C) MET $15 \%$, D) DMSO 10\%, E) DMSO 15\%. 

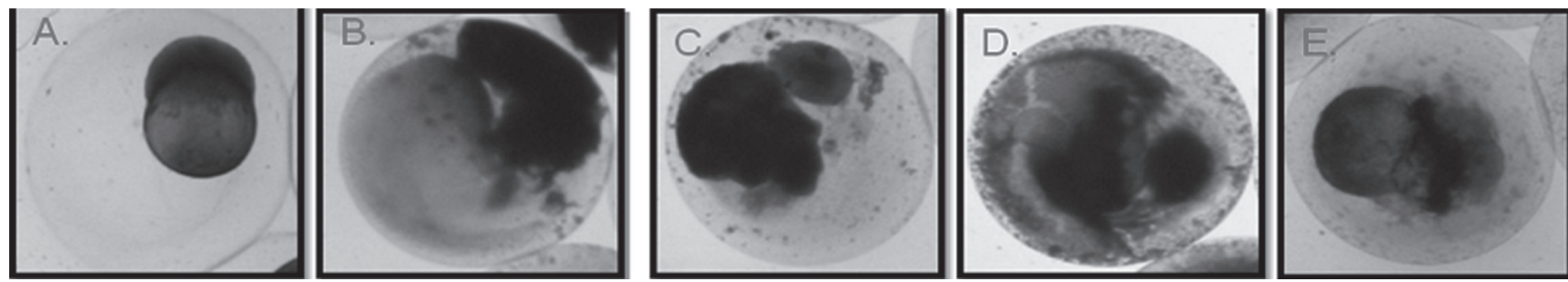

Figura 8. Embriones de yamú (B. amazonicus) en estado de blastulación ( 2 hpf) sometidos a conservación durante 1 hora a $-14{ }^{\circ} \mathrm{C}$ con diferentes tratamientos. A) Grupo control, B) MET 10\%, C) MET 15 \%, D) DMSO 10\%, E) DMSO 15\%.
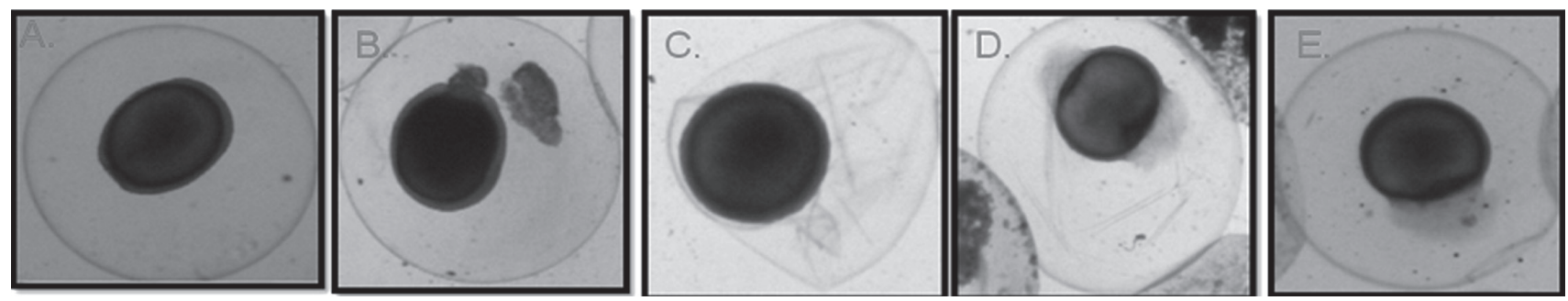

Figura 9. Embriones de yamú (B. amazonicus) en estado de gastrulación ( 6 hpf) sometidos a conservación durante 1 hora a $-14{ }^{\circ} \mathrm{C}$ con diferentes tratamientos. A) Grupo control, B) MET 10\%, C) MET 15 \%, D) DMSO 10\%, E) DMSO 15\%.
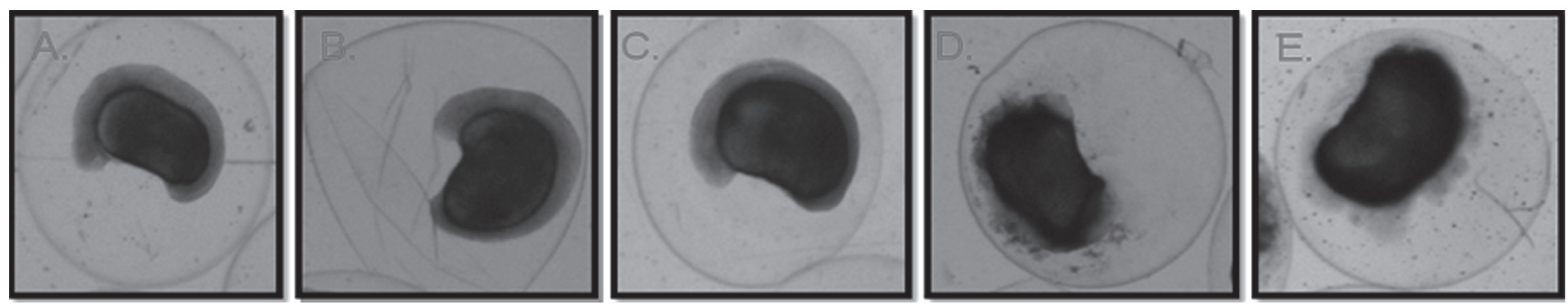

Figura 10. Embriones de yamú (B. amazonicus) en estado de segmentación-faringulación ( 8 hpf) sometidos a conservación durante 1 hora a $-14{ }^{\circ} \mathrm{C}$ con diferentes tratamientos. A) Grupo control, B) MET 10\%, C) MET $15 \%$, D) DMSO 10\%, E) DMSO 15\%.

hpf; discoblástula a las 2 hpf; gástrula a las 5 hpf; organogénesis a las 7 hpf; eclosión a las 12.5 hpf) (Arias, 2006) y con gran cantidad de saco vitelino, lo cual podría impedir la penetración de los crioprotectores (Lopes et al., 2011). Así mismo, en la cachama blanca ( $P$. brachypomus) una especie nativa de la región de la Orinoquia Colombiana, se evaluaron tres estadios embrionarios 1, 6 y $10 \mathrm{hpf}$, observándose mejores porcentajes de viabilidad y sobrevivencia en embriones de 10 hpf, expuestos a DMSO $12 \%$ y MET 10, 12 y $15 \%$ (Castillo-Losada et al., 2011). Lopes et al. (2011) reporta que el $100 \%$ de los embriones de pacú (Piaractus mesopotamicus) sometidos a MET $10 \%$ con 1.4 y $5.2 \mathrm{hpf}$ presentaron menores porcentajes de viabilidad y eclosión con respecto a los embriones que tenían un desarrollo mayor a 8 y 13.3 hpf.

Una de las especies que ha servido como modelo de experimentación a nivel mundial para trabajos de crioconservación es el pez cebra (Danio rerio) en el cual se han evaluado las respuestas a diferentes agentes crioprotectores como el DMSO, 1-2 Propanediol y MET en donde los mejores porcentajes de viabilidades fueron observados en embriones con desarrollo embrionario avanzado de segmentación y aparición de las somitas (Bart, 2000; Lahnsteiner, 2008). Resultados similares fueron observados en el presente estudio donde los embriones de yamú (B. amazonicus) fueron más susceptibles a los procesos de crioconservación durante las etapas iniciales de desarrollo embrionario, siendo más apropiados los estadios más avanzados, 6 y $8 \mathrm{hpf}$ en los que el embrión ya ha finalizado la gastrulación e inicia un proceso de segmentación.

Se ha reportado que en la mayoría de peces la permeabilidad del saco vitelino es prácticamente nula (Hagedorn et al., 1996; Cabrita et al., 2003). Debido a lo anterior, se han orientado estudios para facilitar la entrada de los diferentes crioprotectores a los diferentes compartimentos internos del embrión por medio 
de microinyecciones, decorionización, permeabilización de las membranas y modificación de los mecanismos de intercambio osmótico (Nakatani et al., 2001).

Otra característica importante responsable de estos resultados es la toxicidad generada por los crioprotectores (Bart, 2000). En los estados iniciales de desarrollo (morulación y blastulación), los órganos y mecanismos metabólicos implicados en los procesos de detoxificación se encuentran poco desarrollados siendo los embriones incapaces de contrarrestar la toxicidad de los crioprotectores (Lahnsteiner, 2008). Esto probablemente también fue un factor que influyó en los bajos porcentajes de viabilidad (MET $1.3 \pm 0.5$ y DMSO $0.5 \pm 0.3 \%, 10 \%$ ) embrionaria a las $2 \mathrm{hpf}$ observados en este estudio.

Se han realizado diversas investigaciones evaluando el efecto de los crioprotectores sobre la viabilidad embrionaria en Cyprinus carpio (Dinnye's et al., 1998), Paralichthys olivaceus (Zhang et al., 2005) y Sparus aurata (Cabrita et al., 2006). En embriones de seabream (Pagrus major) de 36h de desarrollo embrionario fueron evaluados cinco crioprotectores internos o permeables: dimetil sulfóxido (DMSO), glicerol (Gly), metanol (Met), 1,2- propilenglicol (PG), etilenglicol (EG) a concentraciones de 5 a 30\% y expuestos durante 10,30 y 60 minutos y dos crioprotectores externos o no permeables polivinilpirrolidona (PVP) y sacarosa durante 10 y 30 minutos. Los crioprotectores menos tóxicos fueron el PG 15\% durante 30 min con una tasa de eclosión de $93.3 \%$ y DMSO con $82.7 \%$. La tasa de eclosión del PVP disminuyó con respecto al aumento en la concentración y tiempo de exposición y con la sacarosa no se presentaron diferencias significativas con respecto a los grupos control (Xiao et al., 2008). En el desarrollo inicial de este estudio se realizaron evaluaciones donde se constató que los crioprotectores con menores efectos negativos en embriones de yamú fueron DMSO y MET a concentraciones de 10 y $15 \%$. Sin embargo, se pudo observar que independientemente del crioprotector empleado el estado de desarrollo embrionario es importante para un éxito adecuado en los procesos de crioconservación. Por lo tanto, es posible afirmar que la toxicidad del crioprotector cambia dependiendo de la especie y el estadio de desarrollo embrionario siendo de gran importancia realizar estudios preliminares para cada especie objeto de estudio, en donde se determinen las concentraciones y los posibles efectos tóxicos de los crioprotectores.

Los cambios morfológicos observados en los embriones sometidos a diferentes crioprotectores se pueden atribuir a varios factores, dentro de los cuales se encuentran implicados elementos como la osmorregula- ción, formación de cristales de hielo y la permeabilidad de las membranas. El aumento excesivo de volumen, luego de un proceso de descongelación y la presencia de concentraciones de crioprotectores intracelulares puede generar que las células embrionarias incorporen agua excesivamente y aumenten de volumen, ocasionando daños estructurales a nivel interno y rompimiento del corion (Karow, 2001). Comparado con otras especies, la cantidad de agua que contienen los embriones de peces es mucho mayor, lo cual juega un papel muy importante en la efectividad del proceso de conservación (Lopes et al., 2011). Por medio de microscopia electrónica se determinó que los embriones de Danio rerio, estaban conformados en un $61 \%$ por agua y el restante $39 \%$ hacia parte del saco vitelino, lo cual puede variar dependiendo del estado de desarroIlo y de la especie (Hagedorn et al., 1996). Otro factor importante de los cambios morfológicos podría ser la disminución del volumen de las células embrionarias durante el proceso de crioconservación, la cual es generada por los crioprotectores que ocasionan una pérdida importante de agua y deshidratación provocando cambios en la composición y estructuras del medio interno del embrión. En este caso, los solutos podrían cambiar la conformación proteica y el metabolismo de los embriones, además la deshidratación favorecería la pérdida de ubicación normal de estructuras citoplásmicas, aproximándose lo suficiente como para permitir reacciones intermoleculares (Karow, 2001).

Por último y no menos importante, se encuentra la formación de cristales de hielo intra-embrionarios lo cual sucede de forma simultánea a la formación de cristales en el exterior, esto puede ser generado por tasas de descenso térmico muy altas con lo cual el agua interna del embrión no logra salir al medio extracelular, formándose pequeños cristales de hielo internamente (Hagedorn et al., 2004). Por otra parte, si la tasa de ascenso térmico durante la descongelación no es lo suficientemente rápida, se puede ocasionar un efecto de recristalización como consecuencia de un nuevo pasaje a través de la temperatura de nucleación, o por crecimiento de los pequeños cristales que pudieran haberse generado durante el enfriamiento. Esto puede producirse además, por un desequilibrio entre la concentración del crioprotector y la tasa de descenso térmico (Kasai et al., 2002). En el presente estudio se utilizaron tasas de congelación de $2.0 \pm 0.7^{\circ} \mathrm{C} / \mathrm{min}$ y de descongelación de $3.5 \pm 1.5{ }^{\circ} \mathrm{C} / \mathrm{min}$ para evitar la formación de dichos cristales; sin embargo, probablemente estas tasas no fueron suficientes para garantizar un ingreso total de los crioprotectores, lo cual aumentó la posibilidad de formación de cristales de hielo. 
A pesar que el presente estudio muestra resultados satisfactorios de eclosión en embriones de 8 horas de desarrollo con MET 15\% (54.1 $\pm 4.1 \%)$, deben realizarse más investigaciones en el área de la crioconservación o conservación a temperaturas de congelación embriones de peces nativos, que evalúen protocolos que permitan porcentajes de viabilidad y eclosión de las larvas en periodos de tiempo más prolongados y disminuyan los criodaños generados por los cambios de osmorregulación y formación de cristales de hielo.

\section{Agradecimientos}

Los autores agradecen a todas las personas que apoyaron el desarrollo experimental del presente trabajo, especialmente a Katherine Betancourt, Jefferson Valderrama, Wilson Corredor Santamaría y Andrés Ramírez Saray y el apoyo logístico del Instituto de Acuicultura de la Universidad de los Llanos.

\section{Referencias}

Arias CJA, Estado actual del conocimiento sobre el yamú, Brycon amazonicus. Revista Colombiana de Ciencias Pecuarias, 2006; 19(2): 125-133

Bart A. 2000. New Approaches in Cryopreservation of Fish Embryos, En: Tiersch TR, Mazik PM (Editores). Cryopreservation in aquatic species. World Aquaculture Society, Baton Rouge, USA, pp. 179-187.

Cabrita E, Robles V, Chereguini O, de Paz P, Anel L, Herráez MP. Dimethyl sulfoxide influx in turbot embryos exposed to a vitrification protocol. Theriogenology, 2003; 60(3): 463-473.

Cabrita E, Robles V, Wallace JC, Sarasquete MC, Herráez MP. Preliminary studies on the cryopreservation of gilthead seabream (Sparus aurata) embryos. Aquaculture, 2006; 251: 245-55.

Castillo-Losada E, Cruz-Casallas PE, Medina-Robles VM. 2011. Conservación de embriones de Cachama blanca (Piaractus brachypomus) como estrategia para su preservación y producción en épocas no reproductivas. En: Memorias Jornada XVII de Acuicultura IALL. ISBN: 978-958-8594-47-7. VillavicencioColombia, p. 33-42.

Cerdá J. Mecanismos fisiológicos durante la hidratación del huevo de teleósteos: hacia el desarrollo de nuevos métodos de criopreservación. Boletín Instituto español de Oceanografía, 2002; 18(1-4): 145-152.

Cruz-Casallas PE, Medina-Robles VM, Velasco-Santamaría YM, Protocolo para la crioconservación de semen de yamú: Brycon amazonicus Spix \& Agassiz 1829. Revista Colombiana de Ciencias Pecuarias, 2006a;19(2): 146-151.

Cruz-Casallas PE, Medina Robles VM, Velasco Santamaría YM. Evaluación de diferentes crioprotectores para la crioconservación de espermatozoides de yamú (Brycon amazonicus). Revista Colombiana de Ciencias Pecuarias, 2006b; 19(2): 152-159.
Dinnye's A, Urbanyi B, Baranyai B, Magyary I. Chilling sensitivity of carp (Cyprinus carpio) embryos at different developmental stages in the presence or absence of cryoprotectants: work in progress. Theriogenology, 1998; 50: 1-13.

Hagedorn MM, Kleinhans FW, Wildt DE, Rall, WF. Chill Sensitivity and Cryoprotectant Permeability of Dechorionated Zebrafish Embryos, Brachydanio rerio. Cryobiology, 1997; 34(3): 251-263.

Hagedorn M, Peterson A, Mazur P, Kleinhans FW. High ice nucleation temperature of zebrafish embryos: slow-freezing is not an option. Cryobiology, 2004; 49: 181-189.

Hagedorn MM, Hsu EW, Pilatusi U, Wildt DE, Rall WF, Blackband SJ. Magnetic resonance microscopy and spectroscopy reveal kinetics of cryoprotectant permeation in a multicompartmental biological system. Proceedings of the National Academy of Sciences, 1996; 93: 7454-7459.

Karow AM. Cryobiology. 2001. For mammalian embryologist. Xytex Cryo International 2001; 18 de agosto de 2012. URL: http:// www.xytexinternational.com/pdf/crybiology.pdf.

Kasai M, Ito K, Edashige K. Morphological appearance of the cryopreserved mouse blastocyst as a tool to identify the type of cryoinjury. Human Reproduction, 2002; 17(7): 1863-1874.

Kawayama S. Highly efficient vitrification for cryopreservation of human oocytes and embryos: The Cryotop method. Theriogenology, 2007; 67(1): 73-80.

Lahnsteiner $F$. The effect of internal and external cryoprotectants on zebrafish (Danio rerio) embryos. Theriogenology, 2008; 69(3): 384-396.

Lopes TS, Romagosa E, Streit JD, Ribeiro RP, Digmayer M. Cooling of Pacú (Piaractus mesopotamicus) embryos at various stages of development for 6 or 10 hours. Theriogenology, 2011; 75(3): 570-576.

Medina-Robles VM, Velasco-Santamaría YM, Cruz-Casallas PE. Efecto del volumen de empaque sobre la tasa de congelación-descongelación y la fertilidad de semen crioconservado de yamú (Brycon amazonicus). Archivos de Medicina Veterinaria, 2007; 39(3): 229-237.

Nakatani K, Agostinho AA, Baumgartner G, Bialetzki A, Sanches PV, Makrakis MC, Pavanelli CS. 2001. Ovos e larvas de peixes de água doce: desenvolvimento e manual de identificação. 1‥ ed. Eduem, Maringá, p.378.

Niemann H. Cryopreservation of ova and embryos from livestock: Current status and research needs. Theriogenology; 1991; 35: 109-124.

Paz HM. 2009. Criopreservación de gametos y embriones En: CarriIlo M, Espinosa de los Montero J. (Editores). La reproducción de los Peces, aspectos básicos y sus aplicaciones en Acuicultura. OESA, CSIC, MMAMRN, Madrid- España. p. 475-526.

Pryor JH, Looney CR, Romo S, Kraemer DC, Long CR. Cryopreservation of in vitro produced bovine embryos: effects of lipid segregation and post-thaw laser assisted hatching. Theriogenology, 2011; 75(1): 24-33. 
Velasco-Santamaría YM, Medina-Robles VM, Cruz-Casallas PE. Cryopreservation of yamú (Brycon amazonicus) sperm for large scale fertilization. Aquaculture, 2006a; 256(1-4): 264-271.

Velasco-Santamaría YM, Corredor-Santamaría W, Cruz-Casallas PE. Efectos del sistema de conservación sobre la fertilidad de oocitos de Yamú (Brycon amazonicus) durante cortos períodos de almacenamiento. Revista Colombiana de Ciencias Pecuarias, 2006b; 19(2): 167-174.

Zhang T, Rawson DM. Studies on chilling sensitivity of zebrafish (Brachydanio rerio) Embryos. Cryobiology, 1995; 32: 239-246.
Zhang T, Liu X, Rawson D. Effects of methanol and developmental arreston chilling injury in zebrafish (Danio rerio) embryos. Theriogenology, 2003; 59: 1545-1556.

Zhang YZ, Zhang SC, Liu XZ, Xu YJ, Hu JH, Xu YY, Li J, Chen Sl. Toxicity and protective efficiency of cryoprotectants to flounder (Paralichthys olivaceus) embryos. Theriogenology, 2005; 63(3): 763-73.

Xiao ZZ, Zhang L, Xu XZ, Liu QH, Li J, Ma DY, Xu SH, Xue YP, Xue QZ. Effect of cryoprotectants on hatching rate of red seabream (Pagrus major) embryos. Theriogenology, 2008; 70: 1086-1092 